\title{
e-Phaïstos
}

e-Phaïstos

Revue d'histoire des techniques / Journal of the history

of technology

IV-2 | 2015

Patrimoines de l'eau

\section{Les ciel-ouverts d'Alger, un savoir-faire importé}

Glass roof overlying stairwells of Algiers, an imported know-how

\section{Asma Hadjilah}

\section{(2) OpenEdition}

Journals

Édition électronique

URL : http://journals.openedition.org/ephaistos/818

DOI : 10.4000/ephaistos.818

ISSN : 2552-0741

Éditeur

IHMC - Institut d'histoire moderne et contemporaine (UMR 8066)

Édition imprimée

Date de publication : 1 octobre 2015

Pagination : 112-121

ISSN : 2262-7340

Référence électronique

Asma Hadjilah, "Les ciel-ouverts d'Alger, un savoir-faire importé », e-Phaïstos [En ligne], IV-2 | 2015,

mis en ligne le 30 novembre 2016, consulté le 10 décembre 2020. URL : http://

journals.openedition.org/ephaistos/818; DOI : https://doi.org/10.4000/ephaistos.818 


\section{Les ciel-ouverts d'Alger, un savoir-faire importé}

Durant l'occupation française, Alger connaît l'introduction de nouvelles techniques de construction; conséquence de la présence dans la ville d'un nombre important d'immigrants européens, parmi lesquels figurait une main d'œuvre essentiellement française et espagnole ${ }^{1}$. La ville adopta ainsi des typologies architecturales répandues alors en Europe. À titre d'exemple, on opta pour la construction d'escaliers à la française, de planchers suivant des procédés expérimentaux alliant fer et brique de terre, ou encore pour la construction de verrières ${ }^{2}$.

Ces dernières furent largement utilisées au niveau des cages d'escalier ; elles étaient désignées, dans le dossier de permis de construire, sous le nom de « ciel vitré ». Dans sa Notice sur la construction de maisons au XIXe siècle à Marseille, l'architecte V. Leroy les nommait «ciel-ouvert» et les définissait comme étant " des ouvertures pratiquées dans la toiture des maisons pour donner le jour aux escaliers »3. Du point de vue constructif, les systèmes d'assemblage utilisés pour ce type de verrières s'apparentaient à ceux employés pour les petits fers, décrits par l'architecte E. Barberot dans le Traité pratique de serrurerie publié en $1888^{4}$.

Les ciel-ouverts d'Alger présentent un intérêt certain : d'une part, ils se révèlent être une solution

\author{
Asma Hadjilah \\ Architecte, \\ Maître assistante chercheuse \\ Ecole Polytechnique d'Architecture et \\ d'Urbanisme / EPAU d'Alger, Algérie
}

innovante. Ils ont comme spécificité de permettre un éclairage naturel des cages d'escalier, ces dernières étant le plus souvent construites au milieu ou en fond de parcelle dans des lieux sombres. D'autre part, ils témoignent de l'usage de nouvelles techniques et de nouveaux matériaux dans le domaine de l'architecture domestique. Aujourd'hui, on peut constater l'existence, dans les premiers quartiers européens d'Alger, d'un ensemble de ces ciel-ouverts qui permet d'apprécier aussi bien la diversité et la variété des formes que les techniques d'assemblage.

Les origines des ciel-ouverts algérois sont peu connues ; cela est dû principalement à l'absence de recherches menées sur les techniques de construction apparues à Alger durant l'occupation française. Néanmoins, la présence de ce type de verrières pourrait témoigner de la circulation de modèles d'un continent à un autre, favorisée par le contexte colonial du XIXe siècle. Ainsi, les cielouverts furent probablement copiés sur des modèles métropolitains, car il existait à cette époque une activité importante d'importation de matériaux de construction de France. De plus, il est à noter que les modèles rencontrés à Alger sont similaires et contemporains à ceux répandus à Marseille. 


\section{Apparition des ciel-ouverts à Alger}

$\mathrm{Au}$ cours de la première moitié du $\mathrm{XIX}^{\mathrm{e}}$ siècle, l'accroissement rapide de la population 5 fut à l'origine de l'apparition des premières maisons européennes : R. Lespès en dénombre 218 élevées dans les rues d'Alger à la fin de l'année $1839^{6}$.

Bien que se déclinant en plusieurs typologies, le modèle de la maison européenne varie peu. Il est possible de le définir comme étant un immeuble élevé de trois ou quatre niveaux, abritant plusieurs appartements desservis par une cage d'escalier. Hormis celles faisant l'angle des rues, ces maisons ont généralement une seule façade donnant sur la rue; c'est probablement pour cette raison que la cage d'escalier est placée au fond de la parcelle, favorisant de ce fait la construction d'un ciel-ouvert afin de l'éclairer.

Par ailleurs, il est possible de constater, notamment grâce à l'annuaire de 1848 , que parmi la population d'origine européenne nouvellement établie à Alger figuraient de nombreux entrepreneurs et artisans du bâtiment tels que: les badigeonneurs, des ferblantiers, des marbriers-sculpteurs, des menuisiers, des serruriers, des charpentiers ; mais aussi des fournisseurs de bois, de faïences et de porcelaines ${ }^{7}$. Cet état de fait témoigne de la présence à Alger d'une main d'œuvre européenne qui pourrait être à l'origine de l'adoption de nouvelles techniques de construction (ex. les planchers et les
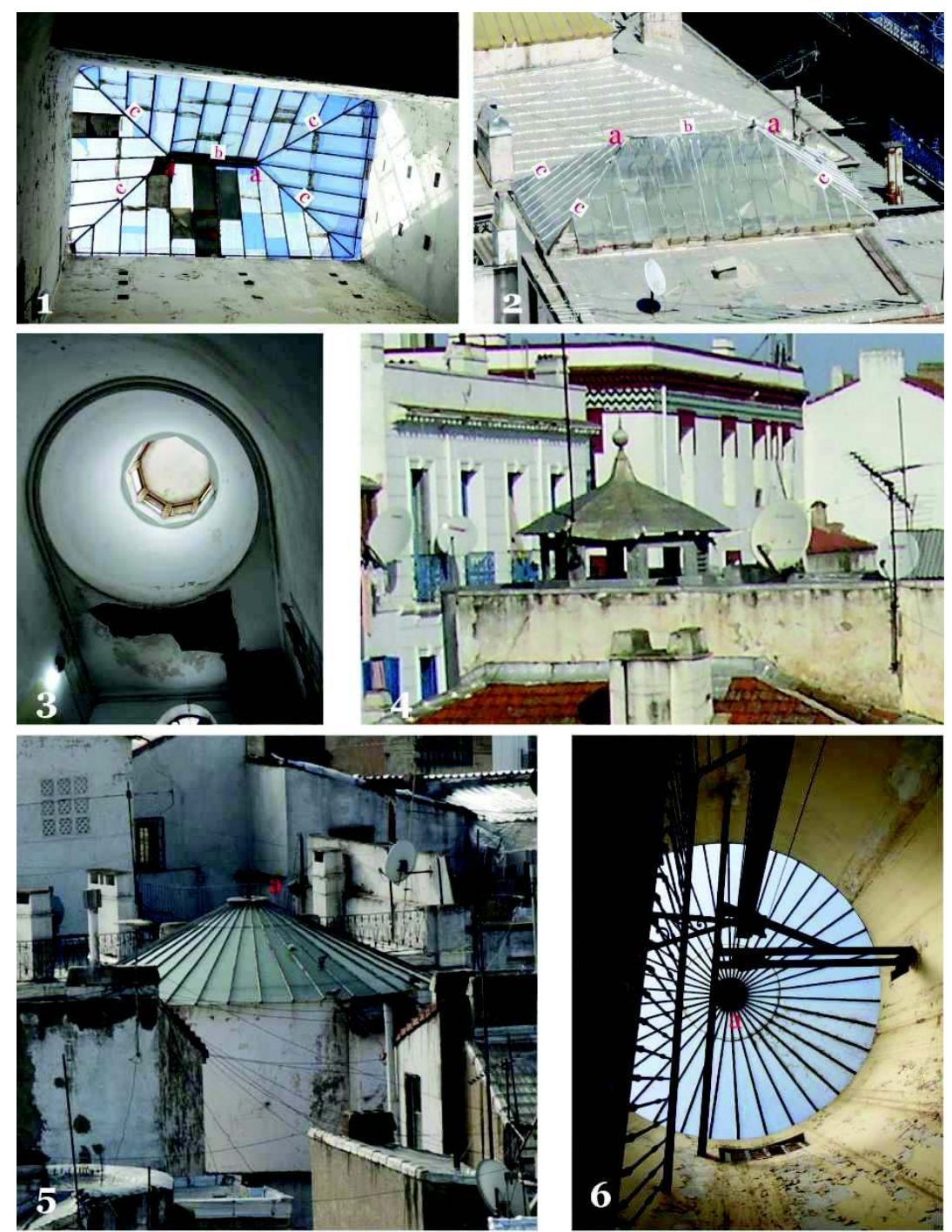

1 ; 2 : Ciel-ouvert à plusieurs plans inclinés, années 1890.

3 ; 4 : Ciel-ouvert à châssis en bois posés verticalement sur un dôme à base circulaire, années 1860. $5 ; 6$ : Ciel-ouvert de forme conique, années 1900

$\mathbf{a}$ : Technique d'assemblage au sommet d'un comble pyramidal à l'aide d'un gobelet ; $\mathbf{b}$ : Panne faîtière ; c : ligne rampante.

Figure 1: Typologies des ciel-ouverts rencontrés à Alger, photographies d'Asma Hadjilah. 
escaliers $^{8}$ ), ou encore de l'introduction de nouveaux éléments architecturaux à l'image des ciel-ouverts.

Les premières maisons européennes construites entre 1835 et $1846^{9}$ sont dotées de ciel-ouverts. La présence de ces derniers en grand nombre justifierait l'existence à cette époque d'une activité d’importation de matériaux manufacturés. En effet, Rozet classe les bois à construction sciés parmi les principaux articles d'importation, et laisse sousentendre leur figuration parmi les produits d'usage chez une population européenne principalement ${ }^{10}$. Outre ces bois ${ }^{11}$, le fer en barre et le plâtre, qui sont également utilisés pour la construction des cielouverts, parviennent dès 1833 de Marseille ${ }^{12}$.

Durant la seconde moitié du XIX $\mathrm{XI}^{\mathrm{e}}$ siècle, la construction de verrières se généralisa pour recouvrir les passages dédiés au commerce, les cours d'immeubles, ou encore les patios des palais et des maisons mauresques. À titre d'exemple, le patio du palais Hassan Pacha, qui servit à partir de 1830 de Palais d'hiver du gouverneur d'Alger ${ }^{13}$, fut couvert en 1875 d'une verrière à plusieurs plans inclinés. Leurs techniques d'assemblage varient peu et s'apparentent à celles des ciel-ouverts.

\section{L'architecture des ciel-ouverts}

À Alger, il est possible d'identifier trois grands types de ciel-ouvert : ceux à un ou plusieurs plans inclinés, ceux de forme conique et ceux à châssis en bois posés verticalement [Figure 1].

Selon V. Leroy, les ciel-ouverts de forme conique ou encore ceux à plans inclinés sont, durant la première moitié du XIX ${ }^{\mathrm{e}}$ siècle, largement utilisé à Marseille ${ }^{14}$; contrairement aux ciel-ouverts à châssis en bois posés verticalement qui furent
Exemples d'escaliers à limons droits :
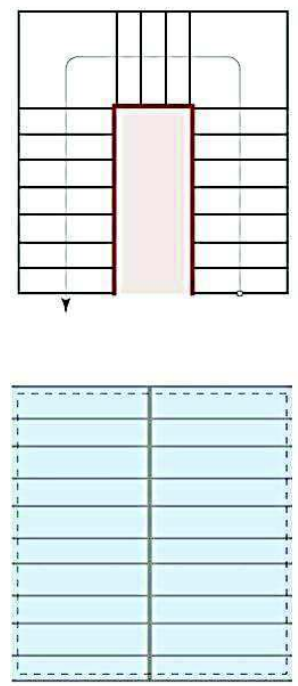

- à deux plans inclinés
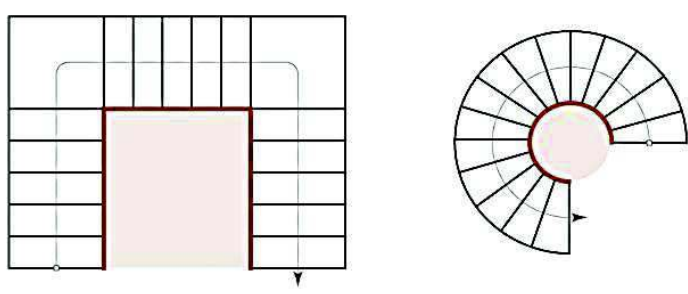

Typologies de ciel-ouverts :

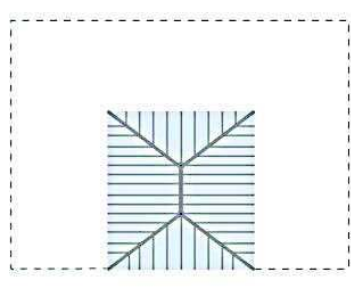

- à quatre plans inclinés

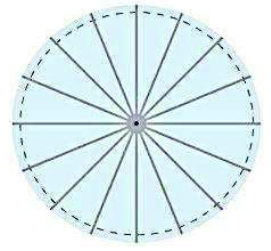

- de forme conique

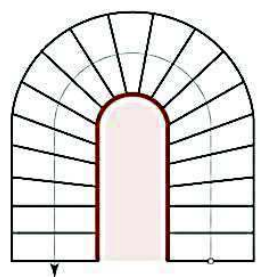

Légende :

Jour

Limon

Figure 2 : Typologies d'escaliers et leurs ciel-ouverts, par Asma Hadjilah. 
bannis, notamment à cause de leur coût élevé $e^{15}$. De même, il n'existe à Alger que peu d'exemples appartenant à ce dernier type; le dispositif nécessaire pour leur recouvrement pourrait donc justifier leur rareté ${ }^{16}$.

Le choix du modèle de ciel-ouverts dépend de la typologie de l'escalier et de ses proportions [Figure 2]: les escaliers à limons ${ }^{17}$ droits sont surmontés de cielouverts à un ou plusieurs plans inclinés. Notons qu'ils recouvrent tout le vide de l'escalier quand ce dernier est de modeste

dimension. En revanche, leur emprise correspond à la taille du jour ${ }^{18}$ de l'escalier quand celui-ci est de grande dimension.

Quant aux escaliers à limons courbes, ils présentent généralement des

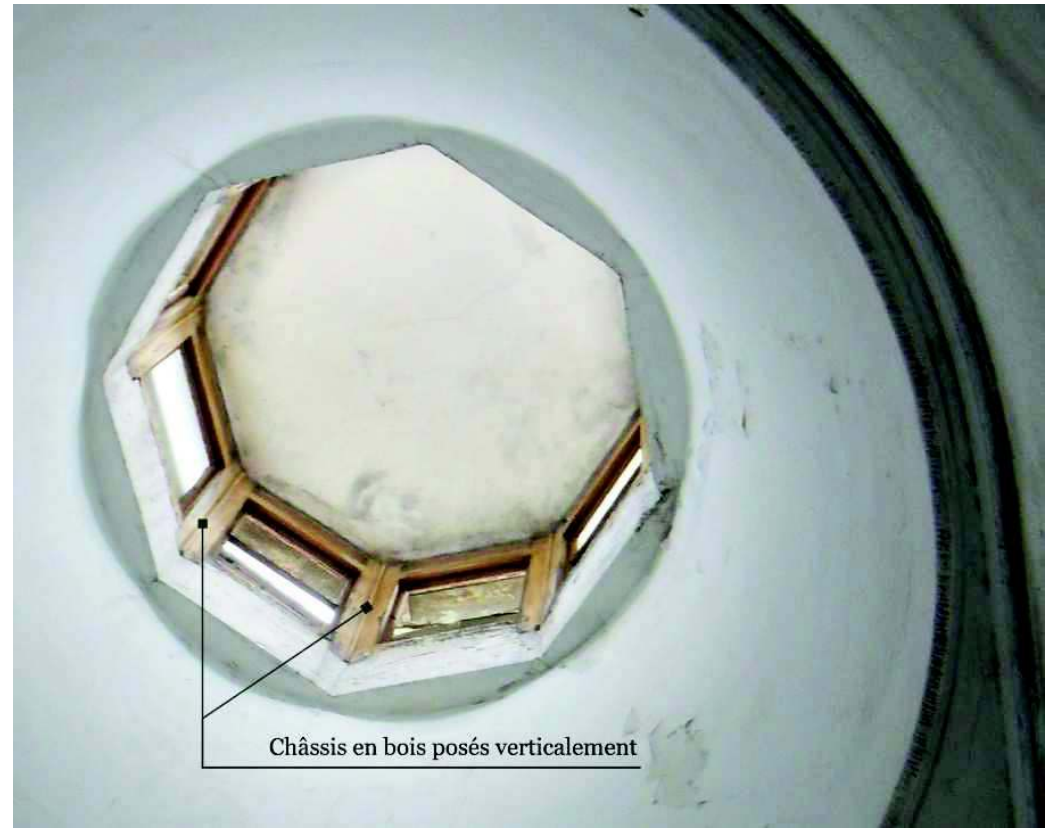

Figure 3 : Châssis en bois posés verticalement d'un ciel-ouvert, photographie d'Asma Hadjilah.

\section{suivants [Figure 4]:}

- Ils doivent être surélevés par rapport au plafond afin de permettre le percement, en dessous, d'ouvertures réduisant l'effet de serre à l'intérieur de la cage d'escalier. Notons que dans le cas où cette dernière est présente sur une de ses parois des ouvertures donnant sur une cour, le ciel-ouvert n'est surélevé que de quelques dizaines de centimètres par rapport au plafond et repose sur une assise en maçonnerie ou en bois.

- Les

barres en fer sur lesquelles reposent les plaques de verre sont à section en $\mathrm{T}$ dont le patin, inférieure, est [Figure 5]. coudé

- Le verre utilisé est un verre double ${ }^{19}$. Les plaques sont posées à joint vif, fixées à l'aide d'un mastic blanc et se superposent sur au moins $10 \mathrm{~cm}$ afin de garantir l'imperméabilité de l'ouvrage ${ }^{20}$. Il est à noter qu'au début du $\mathrm{XX}^{\mathrm{e}}$ siècle, ce type de verre fut remplacé par du verre armé21.

\section{Les techniques d'assemblage}

L’ingéniosité des ciel-ouverts réside dans leur système d'assemblage. Ces derniers devaient en à l'extrémité 


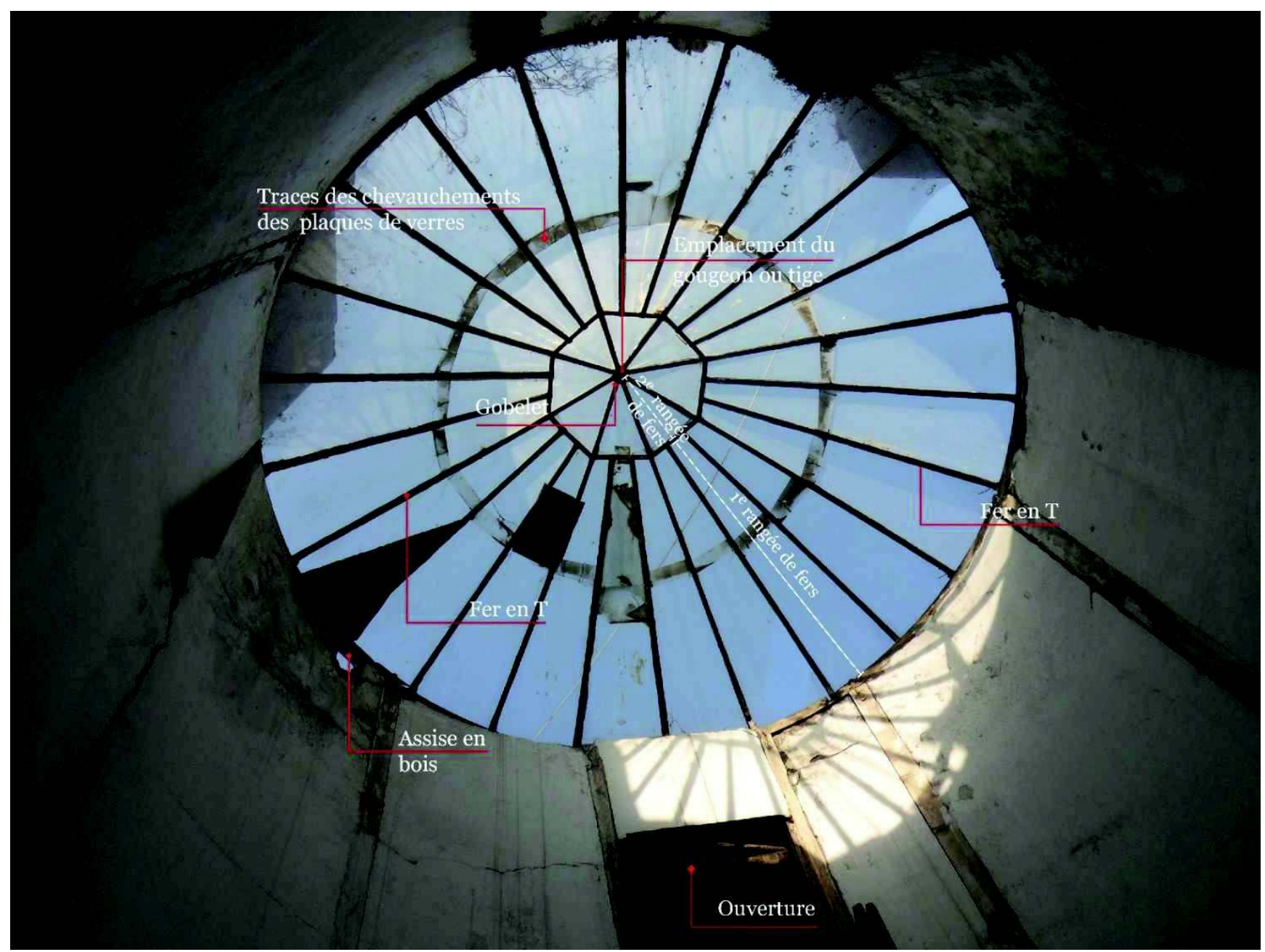

Figure 4 : Identification des éléments constitutifs d'un ciel-ouvert, photographie d'Asma Hadjilah.

effet assurer non seulement la stabilité des ouvrages, mais aussi permettre l'économie de temps et d'argent. D'ailleurs, il est à noter l'absence de rivetage ou d'autre système de montage définitif, afin de rendre possible le remplacement des éléments endommagés.

L'observation des ciel-ouverts d'Alger permet d'identifier certaines des techniques d'assemblage décrites par E. Barberot ${ }^{22}$.

Pour les verrières à un seul plan incliné est employée une série de fers en $\mathrm{T}$ fixés directement sur une base maçonnée ou en bois, à l'aide de fers de même section qui leurs sont perpendiculaires. Quand le ciel-ouvert est de grande dimension, 
l'emploi de fers intermédiaires devient nécessaire afin de donner plus de solidité à l'ouvrage. Les patins et les crêtes des fers sont entaillés de façon à garantir leur emboîtement ; de cette manière le tout ne semble former qu'une seule pièce. Cette technique est également employée dans les autres modèles [Figure 6].

Les ciel-ouverts à quatre plans inclinés ou de forme conique nécessitent l'emploi d'un système d'assemblage plus complexe [Figure 7] :

Pour les verrières de forme conique, qui sont constituées d'une ossature composée de fers en T se joignant au sommet, on utilise la technique d'assemblage au sommet d'un comble pyramidal à l'aide d'un gobelet. Les modèles de ciels-ouverts algérois permettent d'observer la présence de ce dernier qui consiste, d'après E. Barberot, en un anneau en fer plat et épais ${ }^{23}$. Sur cette pièce sont pratiquées des mortaises ou fentes ${ }^{24}$, chacune d'elles accueille un fer dont l'extrémité est découpée en forme de crochet.

L'auteur indique également la présence de clavette fixant chacun des fers à l'intérieur du gobelet. Quand le nombre de fers n'est pas important, les tampons du gobelet peuvent se substituer aux clavettes. Par ailleurs, le diamètre du gobelet est déterminé suivant le nombre des fers qui viennent s'y assembler. Afin de réduire sa taille, il fut d'usage d'employer deux rangées de fers de sorte à restreindre le nombre au sommet [Figure 4].

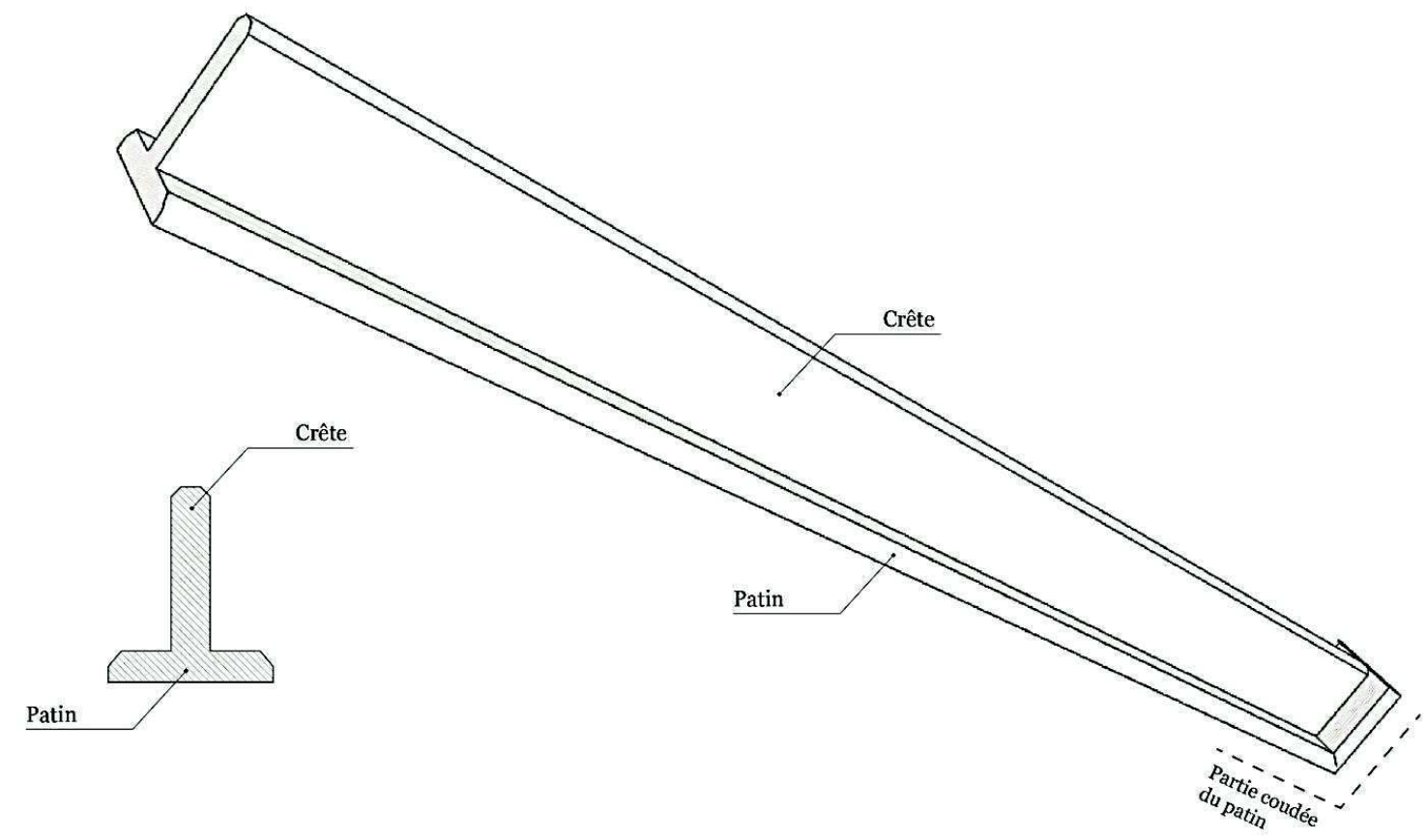

Figure 5 : Fer en T, éléments constitutifs, par Asma Hadjilah. 
Enlaille a $\frac{1}{2} \mathrm{feP}$

Fig.16.
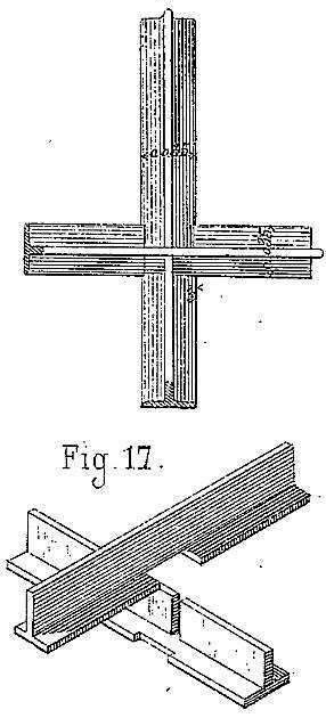

Figure 6 : Assemblage de fers en T moitié par moitié, par E. Barberot, Extrait de Pl 14-15, Nouvelles annales de la construction.

Pour les ciels-ouverts à quatre plans inclinés, l'assemblage s'apparente à celui des charpentes à quatre pentes [Figure 1]. En effet, il est possible d'identifier à leur sommet, un fer horizontal que l'on peut désigner comme étant une panne faîtière ; chacune de ces deux extrémités se trouve un dispositif à gobelet qui permet de joindre les fers délimitant trois pentes ou plans. Le nombre de fers à assembler étant ainsi restreint pour chaque gobelet, l'emploi de clavettes n'est pas nécessaire. Par ailleurs, les fers appartenant à un même plan s'accrochent sur les fers des lignes rampantes ${ }^{25}$ formées par l'intersection de deux plans.

\section{Conclusion :}

Ainsi, les ciel-ouverts d'Alger attestent de la présence d'un savoir-faire importé qui se traduit par l'emploi de matériaux nouveaux et l'adoption de techniques d'assemblage ingénieuses répandues en Europe. Ils constituent de plus un précieux témoignage de l'application du fer dans l'architecture domestique algéroise du XIX ${ }^{\mathrm{e}}$ siècle.

Bien qu'ayant été restreinte aux verrières recouvrant les cages d'escalier, l'étude menée permet de révéler que l'utilisation du fer dans ce

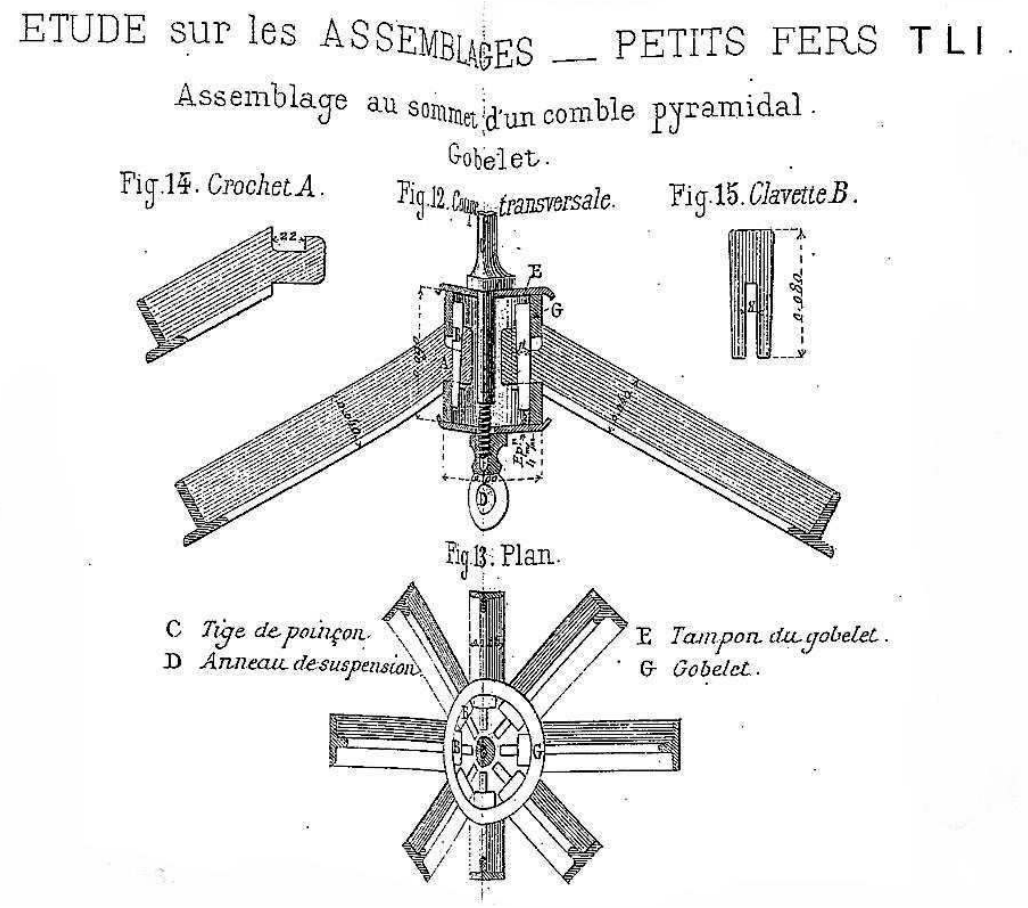

Figure 7: Assemblage de fers en Tau sommet d'un comble pyramidal, par E. Barberot, Extrait de Pl 14-15, Nouvelles annales de la construction. 
type de constructions légères s'accompagnait d'une rationalisation des éléments constructifs. Celle-ci se traduit notamment par l'emploi courant, non seulement du gobelet, mais aussi de fers de même section; elle eut, sans nul doute, pour objectif de réduire les coûts et d'assurer une rapidité et une simplicité d'assemblage.

Par ailleurs, le large emploi des verrières à partir de la seconde moitié du XIX ${ }^{\mathrm{e}}$ siècle, non seulement dans les nouvelles constructions, au niveau des cours et des passages, mais aussi comme système de recouvrement des anciens patios mauresques, atteste une adaptation de techniques d'assemblage européennes à un type de construction qui en est à l’origine dépourvue.

L'étude menée sur les ciel-ouverts gagnerait à être élargie aux autres modèles de verrières, comme ceux recouvrant les anciens patios mauresques. Bien qu'adoptant les mêmes principes d'assemblage, ces verrières sont dotées de caractéristiques propres qui méritent d'être révélées.

1 LESPES René, Alger. Études de géographie et d'histoire urbaine, Paris, Felix Alcan, 1935, p. 499-500.

2 Voir: GUILLERME André, bâtir la ville: Révolutions industrielles dans les matériaux de construction, France Grande-Bretagne (1760-1840), Paris, Champ vallon, 1995. Dès la fin du XVIIIe siècle, ces nouveaux matériaux furent expérimentés, principalement en Grande-Bretagne, dans des ouvrages d'art et édifices militaires. Dans le premier quart du XIXe siècle, leur usage fut généralisé au secteur privé. À titre d'exemple, en France, on abandonna progressivement les planchers en bois au profit des planchers en voutains de briques montés sur des solives en fer (utilisation du système Roussel pour les planchers des $n^{\circ} 4$ et 6 du boulevard des Italiens, Paris).

3 LEROY Victor, Notice sur les constructions des maisons à Marseille au XIXe siècle, Aix-en-Provence, Edisud, 1989, p. 87.

4 Voir aussi : BARBEROT Etienne, «Étude sur les assemblages », Nouvelles annales de la construction, I, 351, mars 1884, p. 3739. Cette étude fut en 1888 incluse dans un ouvrage du même auteur consacré aux constructions en fer. Il fut publié en
Belgique puis en France. Voir: BARBEROT Etienne, Traité pratique de serrurerie. Constructions en fer, Liège, Librairie polytechnique Beauderie \& Cie, 1888.

5 LESPES René, 1935, op. cit., p. 498-499. Voir également : AGERON Charles-Robert, Histoire de l'Algérie contemporaine, Paris, PUF, 1964, p. 20. De la période allant de 1830 à 1840 , l'auteur parle d'une colonisation libre des villes du pays qu'il qualifie d'anarchique.

${ }^{6}$ Afin de les distinguer des maisons mauresques, c'est ainsi que sont désignés les immeubles locatifs construits à partir de 1830 par la nouvelle population européenne.

7 BÉQUET M., L'Algérie en 1848, Paris, Librairie de L. Hachette \& Cie, 1849, p. 481-506.

8 À titre d'exemple, on note l'apparition des planchers en fer à T avec voutains en briques en substitution des planchers traditionnels en rondins de bois. Pour la constitution des planchers traditionnels, voir: MISSOUM Sakina, Alger à l'époque ottomane : la médina et la maison traditionnelle, Alger, INAS, 2003.

9 Elles se situent dans le premier quartier européen d'Alger qui est issu de la restructuration de l'espace intramuros de la médina turque entreprise dès 1830 et qui consista en l'alignement et l'élargissement des voies préexistantes et en la création de nouvelles rues. Voir : JORDI Jean-Jacques, «Alger 1830-1930 ou une certaine idée de la construction de la France", Méditerranée, Tome 89, 2-3-1998, p. 29-34, disponible à l'adresse :

http://www.persee.fr/web/revues/home/prescript/article/medit 0025-8296 1998 num 8923045 , consulté le 9 janvier 2015 .

${ }^{10}$ CARETTE Ernest et ROZET Claude-Antoine, L'Algérie, Paris, Firmin Didot Frères, 1850, p. 302. Voir aussi : LESPES, 1935, op. cit., p. 238. En note $\mathrm{n}^{\circ} 3$ de l'auteur. R. Lespès évoque une ordonnance datant du 11 novembre 1835 autorisant la franchise du bois et des matériaux de construction importés de l'étranger.

${ }^{11}$ Il pourrait s'agir du bois longtemps entreposé au niveau de la place du Gouvernement. Voir : LESPES, 1935, op. cit., p. 221. En note $\mathrm{n}^{\circ} 2$ de l'auteur.

${ }^{12}$ GUIRAL Pierre, Marseille et L'Algérie. 1830 - 1841, Gap, OPHRYS, 1956, p.93.

${ }^{13}$ Pour l'histoire de ce palais, voir : VIDAL-BUE Marion, Villas et plais d'Alger. Du XVII e siècle à nos jours, Paris, Place des victoires, 2012, p. 43-47. 
14 Voir : LEROY, 1989, op. cit., p. 87-89.

${ }_{15}$ De plus, ce modèle ne donne que peu de lumières à la cage d'escalier.

${ }^{16}$ En effet, la construction de ciel-ouverts à châssis en bois posés verticalement nécessite, afin de les protéger de la pluie, la construction d'une toiture en tôle, constituée de versants égaux qui se joignent au sommet en formant une petite pyramide. Le maintien de l'ensemble est garanti par une tige en fer transperçant une sphère. Voir [Figure 1].

${ }^{17}$ Le limon est un membre rampant ou pièce d'appui qui suit la trajectoire de la rampe et qui se trouve à la hauteur des marches. Voir: PEROUSE DE MONTCLOS Jean-Marie, Architecture. Description et vocabulaire méthodique, Paris, Éditions du patrimoine, 2011, p. 368 .

18 Vide autour duquel se développe un escalier tournant.

19 Le verre double est un verre à vitre dont l'épaisseur est comprise entre 3 et 3,5 millimètres.

${ }^{20}$ LEROY, 1989, op. cit., p. 88. Voir aussi : LEMOINE B., L'architecture du fer. France : XIXe siècle, Paris, Champ vallon, 1986, p. 77. Pour la même raison, on prit l'habitude en France, à partir de 1870, de dédoubler les verrières recouvrant les espaces intérieurs en ajoutant un faux plafond horizontal en verre translucide. Toutefois, cette solution n'a pas été rencontrée au niveau des ciel-ouverts algérois.

${ }^{21}$ Le verre armé est un verre renfermant une armature (grille) métallique.

${ }^{22}$ L'étude sur l'assemblage des petits fers de l'architecte E. Barberot rend compte de l'apparition au XIXe siècle de techniques d'assemblage adaptées à chaque type de construction légère. Elle témoigne également d'une généralisation de l'utilisation du fer favorisée par la modernisation des techniques de production. Voir à ce sujet : LEMOINE, 1986, op. cit., p. 38.

23 BARBEROT, 1884, op. cit., p. 38.

24 Trou rectangulaire pratiqué dans une pièce métallique ou en bois afin que celle-ci reçoive une autre pièce.

${ }_{25}$ Désigne le chevron d'arêtier, une pièce de charpente posée obliquement et se trouvant à l'intersection de deux pentes. 


\section{Bibliographie :}

AGERON Charles-Robert, Histoire de l'Algérie contemporaine, Paris, PUF, 1964.

BARBEROT Etienne, «Étude sur les assemblages », Nouvelles annales de la construction, I, 351, mars 1884, p. 37-39.

BARBEROT Etienne, Traité pratique de serrurerie. Constructions en fer, Liège, Librairie polytechnique Beauderie \& Cie, 1888.

BÉQUET M., L'Algérie en 1848, Paris, Librairie de L. Hachette \& Cie, 1849.

CARETTE Ernest et ROZET Claude-Antoine, L'Algérie, Paris, Firmin Didot Frères, 1850.

GUILLERME André, Bâtir la ville : Révolutions industrielles dans les matériaux de construction, France - Grande-Bretagne (1760-1840), Paris, Champ vallon, 1995.

GUIRAL Pierre, Marseille et L'Algérie. 1830 1841, Gap, OPHRYS, 1956.

JORDI Jean-Jacques, " Alger 1830-1930 ou une certaine idée de la construction de la France ", Méditerranée, Tome 89, 2-3-1998, p. 29-34, disponible à l'adresse : http://www.persee.fr/web/revues/home/prescript/ article/medit 0025-

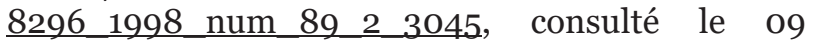
janvier 2015 .

LEMOINE Bertrand, L'architecture du fer. France : XIXe siècle, Paris, Champ vallon, 1986

LEROY Victor, Notice sur les constructions des maisons à Marseille au XIXe siècle, Aix-enProvence, Edisud, 1989.

LESPES René, Alger. Étude de géographie et d'histoire urbaines, Paris, Felix Alcan, 1935

MISSOUM Sakina, Alger à l'époque ottomane: la médina et la maison traditionnelle, Alger, INAS, 2003.
PEROUSE DE MONTCLOS Jean-Marie, Architecture. Description et vocabulaire méthodique, Paris, Édition du patrimoine, 2011.

VIDAL-BUE Marion, Villas et palais d'Alger. Du $X V I I^{e}$ siècle à nos jours, Paris, Place des victoires, 2012. 